\title{
Vibration Mechanism and Compensation Strategy of Magnetic Suspension Rotor System under Unbalanced Magnetic Pull
}

\section{JI ( $\square_{\text {jili198504@hdu.edu.cn ) }}$}

Hangzhou Dianzi University Information Engineering

\section{Xue-qing Ma}

Hangzhou Dianzi University Information Engineering

Zhen-min Chen

ZYEC-EML technologies Co., Ltd.

\section{Original Article}

Keywords: AMB-based motor, Vibration compensation, Unbalanced magnetic pull, Hypothetical reference frame

Posted Date: April 9th, 2021

DOI: https://doi.org/10.21203/rs.3.rs-401924/v1

License: (a) (1) This work is licensed under a Creative Commons Attribution 4.0 International License. Read Full License

Version of Record: A version of this preprint was published at IEEE Access on January 1st, 2022. See the published version at https://doi.org/10.1109/ACCESS.2022.3159675. 


\section{Title page}

\section{Vibration Mechanism and Compensation Strategy of Magnetic Suspension Rotor System under Unbalanced Magnetic Pull}

Li Ji, born in 1984, in 2014, he received his Ph.D. degree in engineering from Nanjing University of Aeronautics and Astronautics, Nanjing, China. His current research interests are in the configuration and control method for high speed magnetic levitation motors.

Tel: +86-0-15952029815; E-mail: jili198504@ hdu.edu.cn

Xue-qing Ma, born in 1996, is currently an assistant professor at Hangzhou Dianzi University Information Engineering School, China. Her research interests include power electronics, motor control and deep learning neural networks.

E-mail: maxueqing@hdu.edu.cn

Zhen-min Chen, born in 1957, is Chairman of ZYEC-EML technologies Co., Ltd., China. Her research interests are in the design and manufacture of PMSM.

E-mail: czm@zyec.com

Corresponding author: Li JI E-mail: jili198504@hdu.edu.cn 


\title{
Vibration Mechanism and Compensation Strategy of Magnetic Suspension Rotor System under Unbalanced Magnetic Pull
}

\author{
$\mathrm{Li} \mathrm{Ji}^{1,2} \cdot$ Xue-qing $\mathrm{Ma}^{1} \cdot$ Zhen-min Chen $^{3}$
}

Received June xx, 201x; revised February xx, 201x; accepted March xx, 201x

(C) Chinese Mechanical Engineering Society and Springer-Verlag Berlin Heidelberg 2017

\begin{abstract}
The existing imbalance compensation strategies for magnetic suspension motors mainly focus on the mass unbalance force, ignoring the effects of the unbalanced magnet pull (UMP). This paper studied the vibration mechanism and compensation strategy of magnetic suspended permanent magnet synchronous motor (PMSM) under the influence of UMP. Firstly, an analytical model of the flux density was established based on equivalent magnetic circuit method, then the analytical expressions of UMP was deduced by Maxwell stress tensor method. Furthermore, an unbalanced compensation method based on hypothetical reference frame (HRF) transformation was proposed. The stability of the closed-loop system with compensation was analyzed based on the complex-coefficients theory. The proposed model and compensation strategy were verified by the simulations and experiments. The results indicate that the proposed compensation strategy can achieve effective suppression of the magnetic rotor vibration.
\end{abstract}

Keywords: AMB-based motor - Vibration compensation • Unbalanced magnetic pull $\bullet$ Hypothetical reference frame

\section{Introduction}

The high speed magnetic suspended motor (the rotor is supported by the magnetic bearing) with high speed (over 10000rpm) can be directly connected to the high speed working machine without acceleration and deceleration

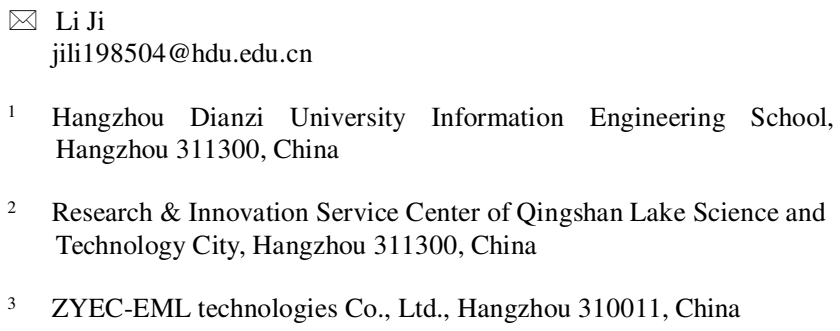

3 ZYEC-EML technologies Co., Ltd., Hangzhou 310011, China

devices. In addition, the magnetic bearings eliminate contact, friction and lubrication system between the stator and the rotor, reducing maintenance costs and extending the life of the motor. The use of AMB-based machine to upgrade the conventional equipment (such as fans, compressors and gas turbines) can increase equipment efficiency significantly [1-4].

Despite their many advantages, magnetic bearings have limited force capacity and imbalance in the rotor mass will cause relatively large synchronous vibration (dynamic eccentricity) when rotor rotates at a high speed [5]. Eccentricity can cause magnetic field distortion, resulting in an unbalanced magnetic pulling force (UMP). In turn, UMP will accelerate the vibration, which may cause instability and collapse of the entire magnetic system. Thus, it is necessary to study the vibration mechanism and compensation strategy of the magnetic suspended rotor under the UMP.

At present, the researches on vibration control of magnetic suspension rotor system mainly focuses on the mass imbalanced force. There are two types of compensation strategies. One strategy is automatic balancing realized mainly through notch filter [6], LMS filter [7], repeated control [8-9], iterative search [10] and sliding mode interference observer [11]. The purpose of this method is to prevent the susceptor vibration caused by the unbalanced force, but the eccentricity of the rotor may increase, which will result in an increase in the unbalanced magnetic pull. Positive feedback between the unbalanced force and the eccentricity could cause the instability of the magnetic suspension system.

The other compensation strategy is to force the rotor to rotate about its geometric axis for better rotation accuracy. Nonami et al. [12] proposed a feedforward 
unbalanced compensation algorithm based on an iterative approximation, which can realize the identification and suppression of interference signals without the system model. Jiang et al. [13] reduced the variable search time for the unbalanced mass independent of the rotational speed by a Fourier iterative search method. Mao et al. [14] proposed a variable step size iterative search algorithm to improve the identification speed. Wang et al. [15] realized unbalanced compensation control by applying an unbalanced coefficient identification. Shi et al. [16] conducted the minimum displacement and minimum current compensation, respectively, based on an adaptive filtering LMS algorithm. Song et al. [17] used a variable polarity LMS feedback based on displacement nulling to compensate unbalance of magnetic bearing. Schuhmann et al. [18] improved the stiffness and dynamic performance of the magnetic bearings through Kalman filtering and LQG state feedback controller. Han et al. [19] applied a repetitive control method to the control system of magnetic bearings, and the vibration of the rotor was effectively reduced.

For the unbalanced compensation strategies, the researches mainly focus on the mass imbalance force. The effects of the UMP have not been systematically studied. In addition, most of the existing compensation methods apply the adaptive iterative algorithm to learn the rotor unbalance parameters. The contradiction between convergence speed and compensation accuracy is inevitable, which results in vibration compensation only for certain rotor speeds. Therefore, it is difficult to realize vibration suppression over the entire rotor speed range. In this paper, an analytical model of UMP based on the special structure of the AMB-based motor was proposed, and a compensation method based on a hypothetical reference frame (HRF) transformation was developed. Finally, the proposed model and compensation strategy were verified by the simulations and experiments.

\section{Model of the Unbalanced Magnetic Pull}

\subsection{Model of the Eccentric Magnetic Density}

The AMB-based motor has the advantages of high-power density and small size, and it is also a complicated machine that consists of the rotor, magnetic bearing, motor stator, displacement sensor, catcher bearing et al. Moreover, there are complicated eccentricity (both static eccentricity and dynamic eccentricity) during operation. The geometric relationship of the rotor eccentricity can be described in the dual coordinate systems, as shown in Fig. 1.

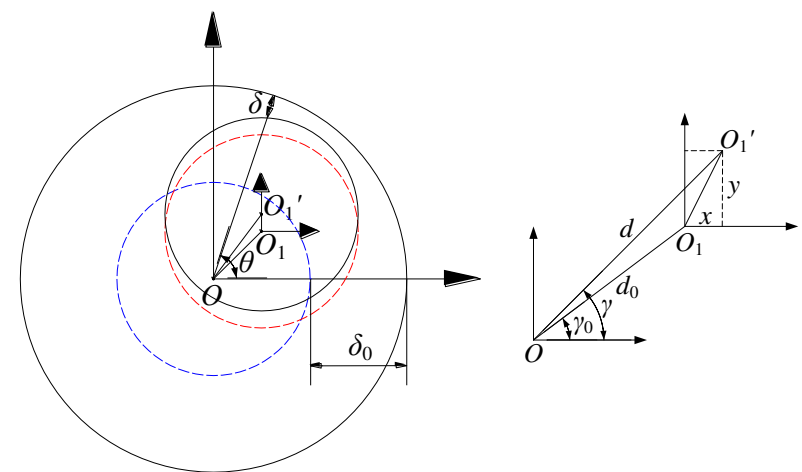

Figure 1 Cross-section of the maglev stator-rotor under air-gap eccentricity

The motor center $O$ is the origin of the $\mathrm{d}-\gamma$ coordinate, and the magnetic bearing center $O_{1}$ is the origin of the x-y coordinate, $O_{1}{ }^{\prime}$ is the geometric center of the rotor. Where $\delta_{0}$ is the nominal air gap length, $d_{0}$ and $\gamma_{0}$ are the static eccentricity and the eccentric angle; $d$ and $\gamma$ is actual eccentricity and eccentric angle of the rotor with both static and dynamic eccentricity.

Since the rotor radius is much larger than the eccentricity, the air gap length at any angle $\theta$ can be approximated as:

$$
\delta(\theta) \approx \delta_{0}-d \cos (\theta-\gamma)
$$

Both the eccentricity and the eccentric angle in the equation can be expressed as a function of $x$ and $y$.

$$
\begin{gathered}
d=\sqrt{\left(d_{0} \cos \gamma_{0}+x\right)^{2}+\left(d_{0} \sin \gamma_{0}+y\right)^{2}} \\
\tan \gamma=\frac{d_{0} \sin \gamma_{0}+y}{d_{0} \cos \gamma_{0}+x}
\end{gathered}
$$

The permeability at any air gap of motor can be expanded into a Fourier series form [20]:

$$
\begin{aligned}
\Lambda(\theta) & =\frac{\mu_{0} \mathrm{~d} S}{\delta(\theta)}=\frac{\mu_{0} \mathrm{~d} S}{\delta_{0}[1-\varepsilon \cos (\theta-\gamma)]} \\
& =\mathrm{d} S \sum_{n=0}^{\infty} \Lambda_{n} \cos n(\theta-\gamma)
\end{aligned}
$$

Where $\mathrm{d} S$ is the magnetic pole area corresponding to the unit angle $\theta, \varepsilon$ is the relative eccentricity, and $\varepsilon=d / \delta_{0}$. The coefficient of $\Lambda_{\mathrm{n}}$ is shown in formula (5).

$$
\Lambda_{n}= \begin{cases}\frac{\mu_{0}}{\delta_{0} \sqrt{1-\varepsilon^{2}}} & (n=0) \\ \frac{2 \mu_{0}}{\delta_{0} \sqrt{1-\varepsilon^{2}}}\left(\frac{1-\sqrt{1-\varepsilon^{2}}}{\varepsilon}\right)^{n} & (n>0)\end{cases}
$$

According to the principle of the permanent magnet motor, the magneto motive force (MMF) is composed of the permanent magnet $F_{\mathrm{j}}$ and Armature $F_{\mathrm{s}}$. Therefore the resultant MMF in the air gap can be expressed as: 


$$
\begin{aligned}
& F(\theta)=F_{\mathrm{j}}(\theta)+F_{\mathrm{s}}(\theta) \\
& =F_{\mathrm{j} 0} \cos (\alpha-p \theta)+F_{\mathrm{s} 0} \cos (\alpha-p \theta-\lambda)
\end{aligned}
$$

Where $p$ is the rotor pole pair number; $F_{\mathrm{j} 0}$ and $F_{\mathrm{s} 0}$ represent the MMF fundamental wave amplitudes of stator and rotor; $\alpha$ is the relative electrical angle between rotor $\mathrm{d}$-axis and stator A-axis. $\lambda$ is the angle between the MMF of stator and rotor (torque angle), which is related to the control strategy of motor.

Ignoring the reluctance of ferromagnetic materials, the expression of the magnetic induction intensity of the air gap with any angle $\theta$ is:

$$
\begin{aligned}
& B(\theta)=\frac{\Lambda(\theta) \cdot F(\theta)}{S} \\
& =F(\theta) \cdot \sum_{n=0}^{\infty} \Lambda_{n} \cos n(\theta-\gamma)
\end{aligned}
$$

\subsection{Analytical Model of Unbalanced Magnetic Pull}

In the case of one pole pair, the radial UMP acting on the rotor due to eccentricity can be obtained based on the Maxwell stress tensor.

$$
\left\{\begin{aligned}
f_{\text {ump_x }} & =\frac{L R \pi}{8 \mu_{0}}\left[k_{1} \cos \gamma+k_{2} \cos (\gamma+\lambda)\right. \\
& +k_{3} \cos (\gamma-\lambda)+k_{4} \cos (2 \alpha-\gamma) \\
& +k_{5} \cos (3 \gamma-2 \alpha)+k_{6} \cos (2 \alpha-\gamma-\lambda) \\
& +k_{7} \cos (2 \alpha-\gamma-2 \lambda)+k_{8} \cos (3 \gamma-2 \alpha+2 \lambda) \\
& \left.+k_{9} \cos (3 \gamma-2 \alpha+\lambda)\right] \\
f_{\text {ump }-y} & =\frac{L R \pi}{8 \mu_{0}}\left[k_{1} \sin \gamma+k_{2} \sin (\gamma+\lambda)\right. \\
& +k_{3} \sin (\gamma-\lambda)+k_{4} \sin (2 \alpha-\gamma) \\
& +k_{5} \sin (3 \gamma-2 \alpha)+k_{6} \sin (2 \alpha-\gamma-\lambda) \\
& +k_{7} \sin (2 \alpha-\gamma-2 \lambda)+k_{8} \sin (3 \gamma-2 \alpha+2 \lambda) \\
& \left.+k_{9} \sin (3 \gamma-2 \alpha+\lambda)\right]
\end{aligned}\right.
$$

Where $\mu_{0}$ is the vacuum permeability and $k_{\mathrm{n}}$ is the coefficient of UMP.

$$
\begin{aligned}
& k_{1}=4 F_{\mathbf{j} 0}^{2} \Lambda_{0} \Lambda_{1}+2 F_{\mathbf{j} 0}^{2} \Lambda_{1} \Lambda_{2}+2 F_{\mathbf{j} 0}^{2} \Lambda_{2} \Lambda_{3}+4 F_{\mathbf{s} 0}^{2} \Lambda_{0} \Lambda_{1} \\
& +2 F_{\mathrm{s0}}^{2} \Lambda_{1} \Lambda_{2}+2 F_{\mathrm{s0}}^{2} \Lambda_{2} \Lambda_{3} \\
& k_{2}=4 F_{\mathrm{j} 0} F_{\mathrm{s} 0} \Lambda_{0} \Lambda_{1}+2 F_{\mathrm{j} 0} F_{\mathrm{s} 0} \Lambda_{1} \Lambda_{2}+2 F_{\mathrm{j} 0} F_{\mathrm{s} 0} \Lambda_{2} \Lambda_{3} \\
& k_{3}=4 F_{\mathrm{j} 0} F_{\mathrm{s} 0} \Lambda_{0} \Lambda_{1}+2 F_{\mathrm{j} 0} F_{\mathrm{s} 0} \Lambda_{1} \Lambda_{2}+2 F_{\mathrm{j} 0} F_{\mathrm{s} 0} \Lambda_{2} \Lambda_{3} \\
& k_{4}=2 F_{\mathbf{j} 0}^{2} \Lambda_{0} \Lambda_{1}+F_{\mathbf{j} 0}^{2} \Lambda_{1} \Lambda_{2}+F_{\mathbf{j} 0}^{2} \Lambda_{2} \Lambda_{3} \\
& k_{5}=2 F_{\mathbf{j} 0}^{2} \Lambda_{0} \Lambda_{3}+F_{\mathbf{j} 0}^{2} \Lambda_{1} \Lambda_{2} \\
& k_{6}=4 F_{\mathrm{j} 0} F_{\mathrm{s} 0} \Lambda_{0} \Lambda_{1}+2 F_{\mathrm{j} 0} F_{\mathrm{s} 0} \Lambda_{1} \Lambda_{2}+2 F_{\mathrm{j} 0} F_{\mathrm{s} 0} \Lambda_{2} \Lambda_{3} \\
& k_{7}=2 F_{\mathrm{s} 0}^{2} \Lambda_{0} \Lambda_{1}+F_{\mathrm{s} 0}^{2} \Lambda_{1} \Lambda_{2}+F_{\mathrm{s} 0}^{2} \Lambda_{2} \Lambda_{3} \\
& k_{8}=2 F_{\mathrm{s} 0}^{2} \Lambda_{0} \Lambda_{3}+F_{\mathrm{s} 0}^{2} \Lambda_{1} \Lambda_{2} \\
& k_{9}=4 F_{\mathrm{j} 0} F_{\mathrm{s} 0} \Lambda_{0} \Lambda_{3}+2 F_{\mathrm{j} 0} F_{\mathrm{s} 0} \Lambda_{1} \Lambda_{2}
\end{aligned}
$$

Obviously, the analytical expression of UMP is very complicated. It includes three fixed-direction force vectors, three double frequency positive sequence components and three double frequency negative sequence components. In order to simplify the formula (8), the magnitude of each component is analyzed under different eccentricity and load rates, and the curves obtained are shown in Figure 2.

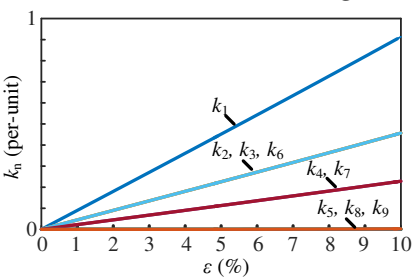

(a)

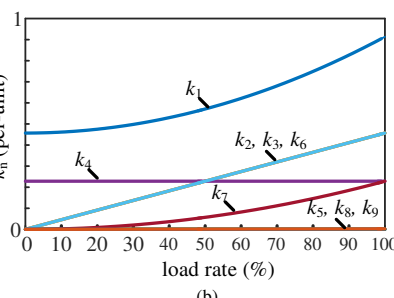

(b)
Figure 2 Unbalanced magnetic pull coefficient against eccentricity and load rate

As shown in the above figure, the amplitudes of the coefficients $k_{5}, k_{8}$, and $k_{9}$ within the entire load range and $10 \%$ relative eccentricity are extremely small. Therefore, $5^{\text {th }}, 8^{\text {th }}$, and $9^{\text {th }}$ items in formula (8) are negligible. The coefficients of the 2 nd and 3rd items are the same and the phase is symmetric about $\gamma$. Thus, they can be merged into one force vector with the first items which is in the same direction as the eccentricity. The $4^{\text {th }}, 6^{\text {th }}$, and $7^{\text {th }}$ items are related to the initial angle $\varphi$. Where, $\varphi$ represents the initial phase difference between the A-axis and the eccentric position.

Moreover, in AMB-based motor, the eccentric angle of the rotor is synchronous with the rotational speed. Therefore, let $\alpha=\omega_{\mathrm{r}} t ; \gamma=\omega_{\mathrm{r}} t+\varphi$. And bring them into formula (8), it can be found that all the items of the UMP in the steady state are positive sequence components with the same frequency as the rotate speed. Therefore, the total UMP exhibits a synchronous rotational force vector, and its amplitude and phase depend on many factors such as the eccentricity $\varepsilon$, the torque angle $\lambda$, and the initial phase difference $\varphi$.

\section{Dynamical Model of AMB-based Motor Under UMP}

Figure 3 shows a schematic diagram of the AMB-based motor. It consists of maglev rotor, motor stator, magnetic bearings, displacement sensor, and catcher bearing. Where $\mathrm{A}$ and $\mathrm{B}$ are the supporting positions of the radial magnetic bearings, $x_{\mathrm{A}}$ and $x_{\mathrm{B}}$ are the displacements corresponding to the radial magnetic bearings in the $x$ 
direction, and $O_{\mathrm{C}}$ is the centroid of rotor.

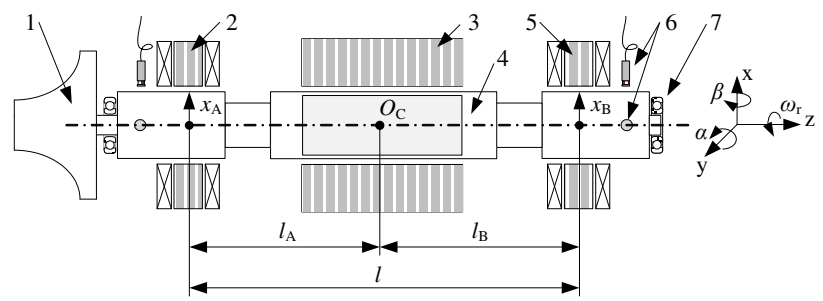

Figure 3 Schematic diagram of AMB-based motor

1. Turbine impeller; 2. Radial magnetic bearing A; 3. Generator stator; 4. Maglev rotor; 5. Radial magnetic bearing B; 6. Radial displacement sensor; 7. Catcher bearing

In the radial direction, the maglev rotor is subjected to a combination of unbalanced mass force, UMP, and control force of magnetic bearing. The control force $f_{\mathrm{mb}}$ can be regarded as a linear function of displacement and control current:

$$
f_{\mathrm{mb}}=k_{\mathrm{d}} \cdot d+k_{\mathrm{i}} \cdot i
$$

Where $d$ is the eccentric displacement of rotor in a certain direction; $i$ is the control current of the magnetic bearing; $k_{\mathrm{d}}$ is the displacement stiffness of the magnetic bearing and $k_{\mathrm{i}}$ is the current stiffness. The control current $i$ is determined by the eccentric displacement and the specific control strategy. For the commonly used PID control strategy, the expression of $i$ is as follows:

$$
i=(-d) \cdot G_{\mathrm{PID}} \cdot G_{\mathrm{A}}
$$

Where, $G_{\mathrm{A}}$ is the transfer function of the power amplifier, which can be considered as a proportional component if the bandwidth of the power amplifier is large enough. The UMP of motor $f_{\text {ump }}$ is shown in formula (8). The unbalance mass $f_{\mathrm{ubm}}$ on the $x, y$ axis can be expressed as:

$$
\left\{\begin{array}{l}
f_{\mathrm{ubm} \_\mathrm{x}}=m\left(e_{\mathrm{p}}-\Delta d\right) \omega_{\mathrm{r}}^{2} \cos \left(\omega_{\mathrm{r}} t+\varphi\right) \\
f_{\text {ubm_y }}=m\left(e_{\mathrm{p}}-\Delta d\right) \omega_{\mathrm{r}}^{2} \sin \left(\omega_{\mathrm{r}} t+\varphi\right)
\end{array}\right.
$$

Where $e_{\mathrm{p}}$ is the distance from the center of mass to the centroid of rotor, $\Delta d=\sqrt{x^{2}+y^{2}}$ is the distance from the centroid to the center of rotation, that is, the dynamic eccentricity, and $\varphi$ is the phase of the unbalance mass. According to the formula $(8 \sim 12)$ and the basic principle of rotor dynamics, the dynamic model of radial AMB system can be established as:

$$
\boldsymbol{M} \ddot{\boldsymbol{x}}+\boldsymbol{G} \dot{\boldsymbol{x}}=\boldsymbol{K}_{\mathrm{d}} \boldsymbol{x}+\boldsymbol{K}_{\mathrm{i}} \boldsymbol{i}-\boldsymbol{E} \boldsymbol{f}_{\mathrm{ub}}
$$

Where $x=\left[x_{\mathrm{A}}, x_{\mathrm{B}}, y_{\mathrm{A}}, y_{\mathrm{B}}\right]^{\mathrm{T}}$ is the displacement of the rotor relative to the equilibrium position of bearing $A$ and $\mathrm{B} ; i$ is the control current matrix of the magnetic bearings; $f_{\mathrm{ub}}$ represents the unbalanced excitation, mainly including the unbalance mass $f_{\text {ubm }}$ and the unbalance magnetic pull $f_{\text {ump }} ; M$ is the generalized mass matrix; $G$ is the gyro matrix; $K_{\mathrm{d}}$ is the displacement stiffness matrix; $K_{\mathrm{i}}$ is the current stiffness matrix; $E$ is the unbalanced excitation response coefficient matrix. Based on the parameters shown in Table 1, setting the speed to $9000 \mathrm{r} / \mathrm{min}$, the eccentricity of the rotor $e_{\mathrm{p}}=10 \mu \mathrm{m}$, and solve formula (13). The simulation results are shown in Figure 4.
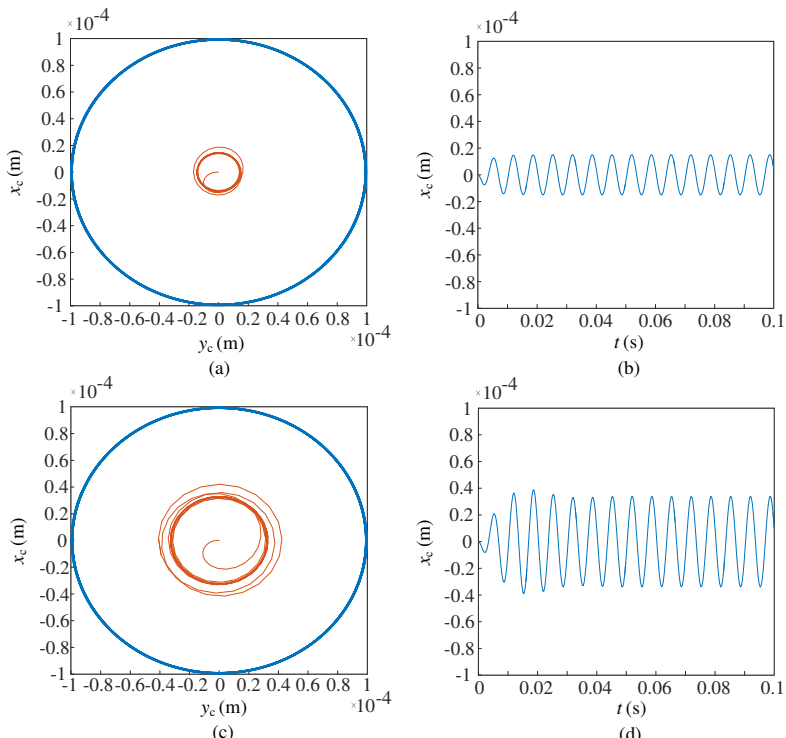

Figure 4 Simulation waveforms of rotor radial vibration with and without unbalanced magnetic pull

Figure 4(a) and (b) are the axial trajectory and vibration waveform of the rotor in $\mathrm{x}$-direction only under the unbalance mass. It can be seen that under current rotation speed, if only the mass unbalance force is applied, the vibration of the rotor is small with only $15 \mu \mathrm{m}$ after stabilization. Figure 4(c) and (d) are the axial trajectory and vibration waveform under both the mass unbalance force and UMP. It can be seen that the vibration increases significantly after taking the UMP into account, and reaches $34 \mu \mathrm{m}$ after stabilization, which is more than double the former. Therefore, the influence of UMP on the vibration of maglev rotor cannot be ignored.

Table 1 Parameters of the prototype

\begin{tabular}{cc}
\hline Parameters & value \\
Rotor quality & $12.5 \mathrm{~kg}$ \\
Rotor equatorial inertial moment & $0.2526 \mathrm{~kg} \cdot \mathrm{m}^{2}$ \\
Rotor polar inertial moment & $0.0096 \mathrm{~kg} \cdot \mathrm{m}^{2}$ \\
air gap length & $5 \mathrm{~mm}$ \\
Stator effective length & $136 \mathrm{~mm}$ \\
Rotor diameter & $56 \mathrm{~mm}$ \\
\hline
\end{tabular}




\section{Unbalanced Compensation Strategy}

\subsection{Control Structure of the Proposed Compensator}

Based on the above analysis and results, it can be seen that eccentricity can cause considerable vibration on the magnetic suspension rotor. In this section, an HRF-based imbalance compensation controller is proposed. The structure used in feedforward compensation is shown in Fig. 5, where $H(s), C(s), A(s), R(s)$ and $K_{s}$ are compensator, master controller, power amplifier, magnetic suspension bearing-rotor and displacement sensor, respectively. The compensator is connected in parallel with the original system controller, and the gain $\varepsilon$ is used to accelerate convergence. Taking the detected displacement error as input, the control quantity of the compensator is added to the output of the original controller.

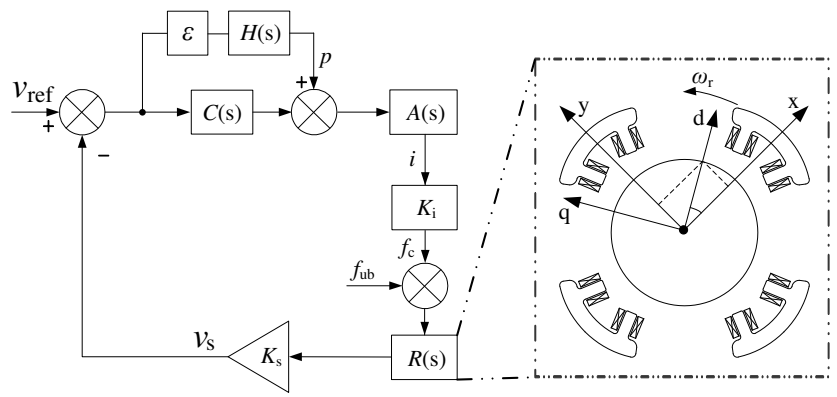

Figure 5 The feedforward compensation control system of the magnetic bearing.

The compensator determines the displacements according to the hypothetical reference frame $d-q$ axis, which rotates synchronously with the actual rotor. Figure 5 shows a cross-sectional view of the rotor, which defines two orthogonal coordinate systems (the $x-y$ and d-q axis).

Fig. 6 shows the internal structure of the compensator, which mainly consists of a coordinate transformation module and an integrator. The principle of the compensator is as follows: two orthogonal rotor displacement errors $v_{\mathrm{x}}$ and $v_{\mathrm{y}}$ are converted into DC signals $\left(v_{\mathrm{d}}, v_{\mathrm{q}}\right)$, and in the ideal case, there is no harmonic component in the DC signal. However, due to the difference in each control loop, the obtained DC signal still has a certain amount of harmonic interference. To further improve the control performance, an integrator is introduced to eliminate the harmonic and amplify synchronous signal. Finally, the control quantities are converted into an AC control signal with a phase shifting through inverse transformation and superimposed into the output of the original controller.

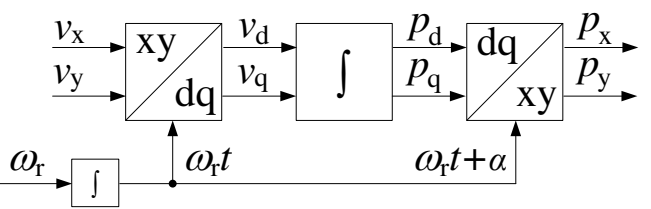

Figure 6 Structure diagram of HRF compensator.

\subsection{Mechanism of Hypothetical Reference Frame}

The HRF transformation matrix $\boldsymbol{T}\left(\omega_{\mathrm{r}} t\right)$ can be expressed as:

$$
\boldsymbol{T}\left(\omega_{\mathrm{r}} t\right)=\left[\begin{array}{cc}
\cos \left(\omega_{\mathrm{r}} t\right) & \sin \left(\omega_{\mathrm{r}} t\right) \\
-\sin \left(\omega_{\mathrm{r}} t\right) & \cos \left(\omega_{\mathrm{r}} t\right)
\end{array}\right]
$$

The two orthogonal signals $\left[v_{\mathrm{x}}, v_{\mathrm{y}}\right]$ can be expressed as $\left[A_{0} \cos \left(\omega_{\mathrm{r}} t+\varphi\right), \quad A_{0} \sin \left(\omega_{\mathrm{r}} t+\varphi\right)\right], \quad$ and after coordinate transformation, one can obtain:

$$
\begin{aligned}
{\left[\begin{array}{l}
v_{\mathrm{d}} \\
v_{\mathrm{q}}
\end{array}\right] } & =\left[\begin{array}{cc}
\cos \left(\omega_{\mathrm{r}} t\right) & \sin \left(\omega_{\mathrm{r}} t\right) \\
-\sin \left(\omega_{\mathrm{r}} t\right) & \cos \left(\omega_{\mathrm{r}} t\right)
\end{array}\right]\left[\begin{array}{c}
A_{0} \cos \left(\omega_{\mathrm{r}} t+\varphi\right) \\
A_{0} \sin \left(\omega_{\mathrm{r}} t+\varphi\right)
\end{array}\right] \\
& =\left[\begin{array}{c}
A_{0} \cos \varphi \\
A_{0} \sin \varphi
\end{array}\right]
\end{aligned}
$$

If the orthogonal signal contains harmonic components $\left[A_{\mathrm{n}} \cos \left(n \omega_{\mathrm{r}} t+n \varphi\right), \quad A_{\mathrm{n}} \sin \left(n \omega_{\mathrm{r}} t+n \varphi\right)\right], \quad$ then after transformation, the signal is transformed as:

$$
\begin{aligned}
{\left[\begin{array}{l}
v_{\mathrm{dn}} \\
v_{\mathrm{qn}}
\end{array}\right] } & =\left[\begin{array}{cc}
\cos \left(\omega_{\mathrm{r}} t\right) & \sin \left(\omega_{\mathrm{r}} t\right) \\
-\sin \left(\omega_{\mathrm{r}} t\right) & \cos \left(\omega_{\mathrm{r}} t\right)
\end{array}\right]\left[\begin{array}{l}
A_{n} \cos \left(n \omega_{\mathrm{r}} t+n \varphi\right) \\
A_{n} \sin \left(n \omega_{\mathrm{r}} t+n \varphi\right)
\end{array}\right] \\
& =\left[\begin{array}{c}
A_{n} \cos \left(n \omega_{\mathrm{r}} t-\omega_{\mathrm{r}} t+n \varphi\right) \\
A_{n} \sin \left(n \omega_{\mathrm{r}} t-\omega_{\mathrm{r}} t+n \varphi\right)
\end{array}\right]
\end{aligned}
$$

The corresponding $n-1$ harmonic component can be obtained, where $n$ represents the harmonic number. These harmonic components can be eliminated by an integrator. Moreover, the integrator has an infinite gain for the DC component. $P$ is the gain when the system reaches steady state and can be expressed as:

$$
\left[\begin{array}{c}
p_{\mathrm{d}} \\
p_{\mathrm{q}}
\end{array}\right]=\left[\begin{array}{c}
P A_{0} \cos \varphi \\
P A_{0} \sin \varphi
\end{array}\right]
$$

The inverse transformation matrix $\boldsymbol{T}_{\text {inv }}\left(\omega_{\mathrm{r}} t\right)$ is expressed as:

$$
\boldsymbol{T}_{\text {inv }}\left(\omega_{\mathrm{r}} t\right)=\left[\begin{array}{cc}
\cos \left(\omega_{\mathrm{r}} t+\beta\right) & -\sin \left(\omega_{\mathrm{r}} t+\beta\right) \\
\sin \left(\omega_{\mathrm{r}} t+\beta\right) & \cos \left(\omega_{\mathrm{r}} t+\beta\right)
\end{array}\right]
$$

Where, $\beta$ is the phase advance supplementary angle, which is used to improve the stability of the system.

After the inverse synchronous rotation transformation, one can have: 


$$
\begin{aligned}
{\left[\begin{array}{l}
p_{\mathrm{x}} \\
p_{\mathrm{y}}
\end{array}\right] } & =\left[\begin{array}{cc}
\cos \left(\omega_{\mathrm{r}} t+\beta\right) & -\sin \left(\omega_{\mathrm{r}} t+\beta\right) \\
\sin \left(\omega_{\mathrm{r}} t+\beta\right) & \cos \left(\omega_{\mathrm{r}} t+\beta\right)
\end{array}\right]\left[\begin{array}{c}
P A_{0} \cos \varphi \\
P A_{0} \sin \varphi
\end{array}\right] \\
& =\left[\begin{array}{c}
P A_{0} \cos \left(\omega_{\mathrm{r}} t+\varphi+\beta\right) \\
P A_{0} \sin \left(\omega_{\mathrm{r}} t+\varphi+\beta\right)
\end{array}\right]
\end{aligned}
$$

\subsection{Stability analysis of Control System}

The proposed HRF compensator uses centralized control for the $\mathrm{x}$ and $\mathrm{y}$ direction. Therefore, this paper introduces the definition of a complex transfer-function, which helps to build a single-input single-output model from the dual-input dual-output system. Then the classical stability criterion can be used in the proposed system.

The HRF compensator with a phase shift in the continuous time domain is shown in Fig. 6. These sinusoidal signals are internally generated by the sine and cosine blocks. The output signals of the compensator can be described as follow:

$$
\begin{array}{r}
p_{\mathrm{x}}(t)=\cos \left(\omega_{\mathrm{r}} t+\beta\right) \int \cos \left(\omega_{\mathrm{r}} t\right) v_{\mathrm{x}}(t) d t+\cos \left(\omega_{\mathrm{r}} t+\beta\right) \int \sin \left(\omega_{\mathrm{r}} t\right) v_{\mathrm{y}}(t) d t \\
+\sin \left(\omega_{\mathrm{r}} t+\beta\right) \int \sin \left(\omega_{\mathrm{r}} t\right) v_{\mathrm{x}}(t) d t-\sin \left(\omega_{\mathrm{r}} t+\beta\right) \int \cos \left(\omega_{\mathrm{r}} t\right) v_{\mathrm{y}}(t) d t \\
p_{\mathrm{y}}(t)=\sin \left(\omega_{\mathrm{r}} t+\beta\right) \int \cos \left(\omega_{\mathrm{r}} t\right) v_{\mathrm{x}}(t) d t+\sin \left(\omega_{\mathrm{r}} t+\beta\right) \int \sin \left(\omega_{\mathrm{r}} t\right) v_{\mathrm{y}}(t) d t \\
-\cos \left(\omega_{\mathrm{r}} t+\beta\right) \int \sin \left(\omega_{\mathrm{r}} t\right) v_{\mathrm{x}}(t) d t+\cos \left(\omega_{\mathrm{r}} t+\beta\right) \int \cos \left(\omega_{\mathrm{r}} t\right) v_{\mathrm{y}}(t) d t
\end{array}
$$

After differentiating the above equations, we can obtain the differential equations of the compensator as.

$$
\begin{aligned}
\ddot{p}_{\mathrm{x}}(t)= & -\omega_{\mathrm{r}}^{2} p_{\mathrm{x}}(t)-\sin \beta \dot{v}_{\mathrm{y}}(t)+\cos \beta \dot{v}_{\mathrm{x}}(t) \\
& -\omega_{\mathrm{r}} \sin \beta v_{\mathrm{x}}(t)-\omega_{\mathrm{r}} \cos \beta v_{\mathrm{y}}(t) \\
\ddot{p}_{\mathrm{y}}(t)= & -\omega_{\mathrm{r}}^{2} p_{\mathrm{y}}(t)+\cos \beta \dot{v}_{\mathrm{y}}(t)+\sin \beta \dot{v}_{\mathrm{x}}(t) \\
& +\omega_{\mathrm{r}} \cos \beta v_{\mathrm{x}}(t)-\omega_{\mathrm{r}} \sin \beta v_{\mathrm{y}}(t)
\end{aligned}
$$

Then, the transfer function from $v(s)$ to $p(s)$ can be derived by Laplace transform, that is

$$
\begin{aligned}
& p_{\mathrm{x}}(s)=\left[\frac{s \cos \beta}{s^{2}+\omega_{\mathrm{r}}^{2}}-\frac{\omega_{\mathrm{r}} \sin \beta}{s^{2}+\omega_{\mathrm{r}}^{2}}\right] v_{\mathrm{x}}(s)-\left[\frac{s \sin \beta}{s^{2}+\omega_{\mathrm{r}}^{2}}+\frac{\omega_{\mathrm{r}} \cos \beta}{s^{2}+\omega_{\mathrm{r}}^{2}}\right] v_{\mathrm{y}}(s) \\
& p_{\mathrm{y}}(s)=\left[\frac{s \cos \beta}{s^{2}+\omega_{\mathrm{r}}^{2}}-\frac{\omega_{\mathrm{r}} \sin \beta}{s^{2}+\omega_{\mathrm{r}}^{2}}\right] v_{\mathrm{y}}(s)+\left[\frac{s \sin \beta}{s^{2}+\omega_{\mathrm{r}}^{2}}+\frac{\omega_{\mathrm{r}} \cos \beta}{s^{2}+\omega_{\mathrm{r}}^{2}}\right] v_{\mathrm{x}}(s)
\end{aligned}
$$

Multiplying the second equation by $j$ and adding to the first equation, the complex transfer function $H(s)$ can be obtained as follows:

$$
\begin{aligned}
& H(s)=\frac{\left(p_{\mathrm{x}}+j p_{\mathrm{y}}\right)}{\left(v_{\mathrm{x}}+j v_{\mathrm{y}}\right)}=\left[\frac{s \cos \beta}{s^{2}+\omega_{\mathrm{r}}^{2}}-\frac{\omega_{\mathrm{r}} \sin \beta}{s^{2}+\omega_{\mathrm{r}}^{2}}\right] \\
& +j\left[\frac{s \sin \beta}{s^{2}+\omega_{\mathrm{r}}^{2}}+\frac{\omega_{\mathrm{r}} \cos \beta}{s^{2}+\omega_{\mathrm{r}}^{2}}\right]
\end{aligned}
$$

In the same way, the complex transfer function of a magnetic suspension bearing-rotor system considered with a gyroscopic coupling effect can be obtained.

$$
R(s)=\frac{(x+y j)}{\left(i_{\mathrm{x}}+i_{\mathrm{y}} j\right)}=\frac{K_{\mathrm{i}}}{M s^{2}-K_{\mathrm{xy}}-j \omega_{\mathrm{r}} G s}
$$

Where, $M$ is equivalent mass of the rotor, $G$ is a coefficient concerning gyroscopic, $K_{\mathrm{xy}}$ is displacement stiffness and $K_{\mathrm{i}}$ is current stiffness.

According to Fig. 5, the characteristic equation of the closed loop system can be expressed as:

$$
1+(C(s)+H(s)) A(s) R(s) k_{\mathrm{s}}=0
$$

$C(\mathrm{~s})$ is a classical PID controller,

$$
C(s)=K_{\mathrm{P}}+\frac{K_{\mathrm{I}}}{s}+\frac{K_{\mathrm{D}} s}{T s+1}
$$

Where, $K_{\mathrm{P}}, K_{\mathrm{I}}$, and $K_{\mathrm{D}}$ are the three parameters of the PID controller, and $T$ is the time constant of the incomplete differential link.

$A(\mathrm{~s})$ is simplified as a gain. Then, the root locus of a magnetic bearing-rotor system with HRF compensator is shown in Fig. 7, where the frequency varies between 0 to $500 \mathrm{~Hz}$. As previously described, the compensation phase $\beta$ plays an important role in the proposed control method for a stable closed-loop system.

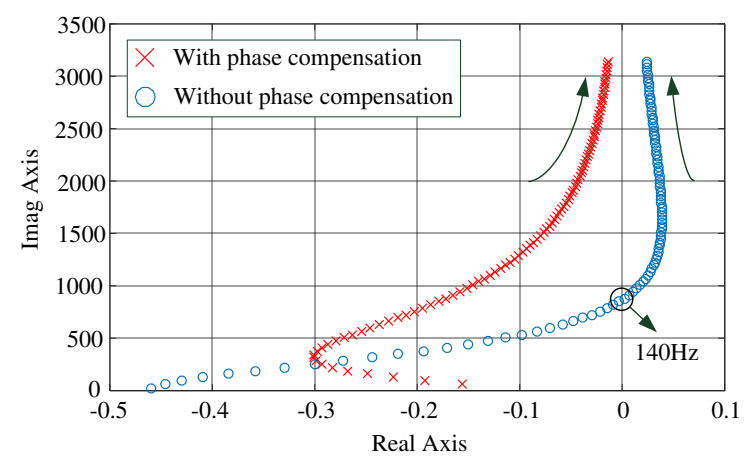

Figure 7 Dominant root locus of the control system with HRF compensator in simulation.

The blue circles with $\beta=0$ indicate that the closed-loop root locus are first moved to the right-half plane at a frequency of $140 \mathrm{~Hz}$. This means that the system is unstable when the rotation frequency is above $140 \mathrm{~Hz}$ with $\beta=0$. The red star line with $\beta=\pi / 3$ shows that the closed-loop root locus is always located at left-half plane, which means the system with the HRF compensator is stable over the entire frequency.

\section{Experimental Results}

In this section, experiments are carried out to verify the previous analysis. The experiments were done with a TMS320F28335 digital controller on a 100kW prototype 
of magnetic suspended permanent magnet motor, as shown in Fig. 8.

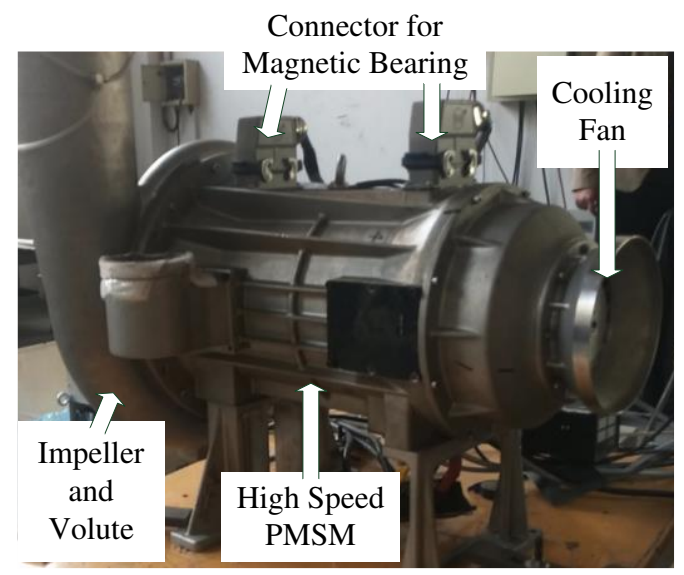

Figure 8 Functional prototype of the magnetic suspended PMSM.

First, a steady-state experiment was carried out, and the proposed compensation strategy was applied to four radial controllers. The displacement signal waveforms are shown in Fig. 9, where channels 1-4 of the oscilloscope correspond to the four radial DOFs of the rotor, respectively. It can be seen that the compensation strategy does not affect the stability at steady-state.

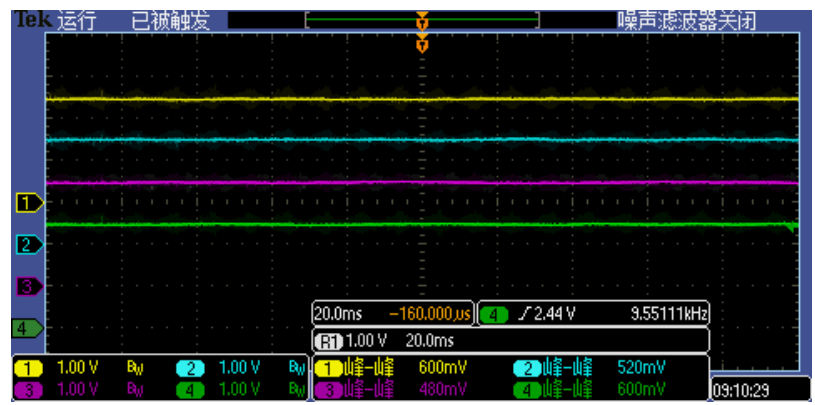

Figure 9 Experimental results of control system in steady state with the proposed method.

Then, a speed-up test was carried out to verify the dynamic performance of the proposed control system. In order to obtain an appropriate compensation phase $\alpha$, the closed-loop spectrum characteristics of the magnetic bearing-rotor system from the unbalanced force to the output displacement are obtained by frequency sweep analysis, as shown in Fig. 10.
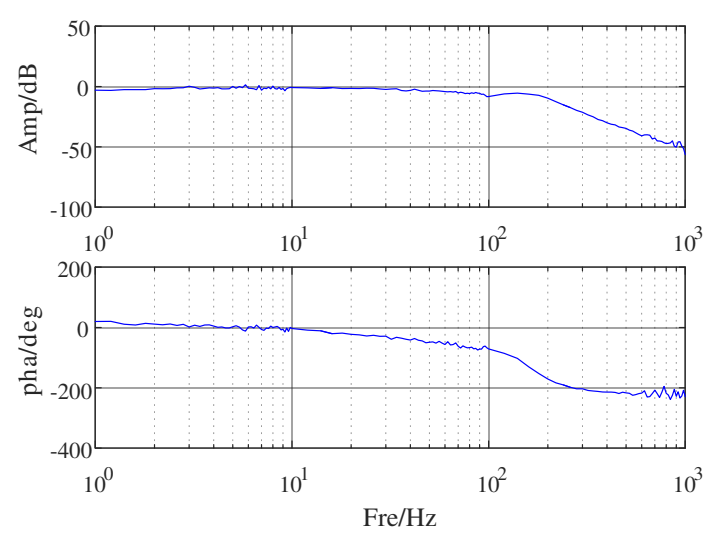

Figure 10 Closed-loop spectrum characteristics of the magnetic bearing-rotor system.

In order to evaluate the performance of the proposed method, a comparison experiment between the HRF compensator and the notch filter was introduced. When the rated speed is $24000 \mathrm{r} / \mathrm{min}(400 \mathrm{~Hz})$, the self-balancing control (notch filter) is applied, and the waveform is shown in Fig. 11 (a). Channels 1 and 3 of the oscilloscope were used to measure the radial control current and its radial displacement signal. It can be seen from Fig. 13 that at this speed, the rotor can be stably suspended with a vibration peak of $460 \mathrm{mV}$. The corresponding displacement sensor has a sensitivity of $60 \mathrm{~m} / \mathrm{V}$ and a vibration amplitude of $27.6 \mu \mathrm{m}$. Since the notch filter can suppress the same frequency signal, there is substantially no same frequency component in the corresponding control current. Applying the proposed HRF compensation strategy under the above condition, the displacement vibration waveform of the rotor at $400 \mathrm{~Hz}$ is shown in Fig. 11 (b). It is worth noting that, compared with the above method, the HRF compensation strategy increases the supporting stiffness of the synchronous frequency signal, and the amplitude of the rotor vibration is significantly decreased by about 14.4 microns, which is only about $52 \%$ of the notch filter control.

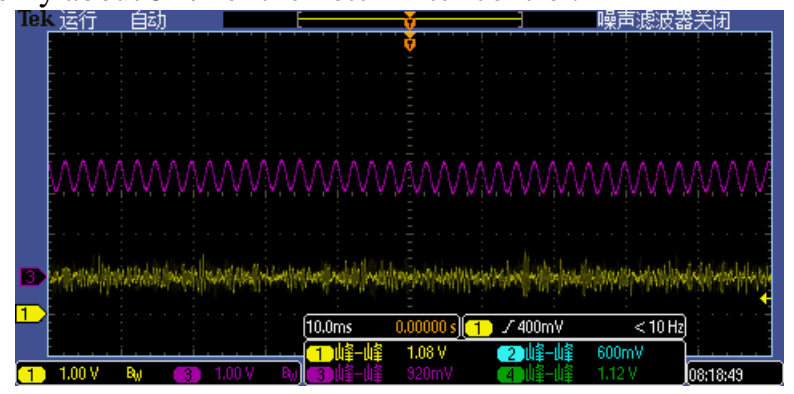

(a) 


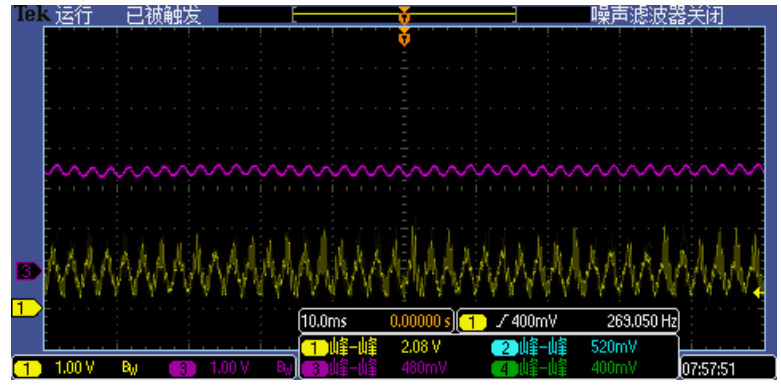

(b)

Figure 11 Experimental results of magnetic bearing-rotor system with the compensator: (a) adaptive notch filter; (b) HRF compensator.

According to the evaluation index $D_{\max } / C_{\min }$ [21] of the vibration level of the magnetic suspension bearing-rotor system defined by ISO 14839-2, the effects of the above two compensation strategies are compared in different working speed ranges, as shown in Fig. 12. Since the two strategies are very close at low rotation speeds, the vibration of a magnetic bearing at a rotation speed of $150 \mathrm{~Hz}-400 \mathrm{~Hz}$ is captured. It can be seen that the proposed compensation strategy is more effective in suppressing the rotor vibration.

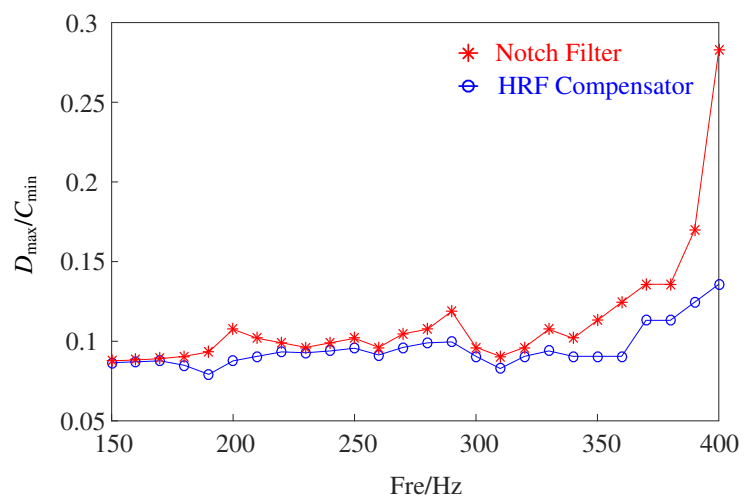

Figure 12 Vibration of the magnetic bearing-rotor system under different rotation speed.

\section{Conclusions}

This paper focused on the high speed magnetic suspension permanent magnet motors. Based on the subdomain method, perturbation method and Maxwell stress tensor method, an analytical model of the unbalanced magnetic pull was established. An unbalanced compensation strategy based on a HRF transformation was developed. The following conclusions can be drawn:

1) Based on the special structure of the high speed magnetic suspension permanent magnet motor, the first order perturbation method was used to establish a complete analytical model of the biased air gap magnetic field and unbalanced magnetic pull. The proposed analytical model is precise, simple and has certain physical meaning.

2) The mathematical model and finite element simulation show that the dynamic eccentricity plays an important role in the magnetic suspension motor system. The unbalanced magnetic pull of the permanent magnet rotor can be represented as a rotating force vector with the same frequency as the rotation speed. There is a linear relationship between the unbalanced magnetic pull and the eccentricity in a small range, and its phase depends on the initial eccentric angle.

3) The compensation phase $\alpha$ is very important for the proposed compensation strategy. By selecting an appropriate compensation phase, the closed-loop system with $\mathrm{HRF}$ compensator can remain stable over the entire frequency.

4) The compensation strategy proposed in this paper does not require complex iterative operation and can suppress the same frequency vibration of the rotor. Compared with the traditional notch filter method, the proposed method has better control effect.

\section{Declaration}

\section{Funding}

This work was supported by the Natural Science Foundation of Jiangsu Province (Grant No. BK20190491).

\section{Availability of data and materials}

The datasets supporting the conclusions of this article are included within the article.

\section{Authors' contributions}

The author' contributions are as follows: Li Ji wrote the manuscript; Xue-qing $\mathrm{Ma}$ and Zhen-min Chen assisted with sampling and laboratory analyses.

\section{Competing interests}

The authors declare no competing financial interests.

\section{Consent for publication}

Not applicable

\section{Ethics approval and consent to participate}

Not applicable 


\section{References}

[1] C. Di, I. Petrov, J. Pyrhönen, et al, "Unbalanced Magnetic Pull Compensation With Active Magnetic Bearings in a 2 MW High-Speed Induction Machine by FEM," IEEE Transactions on Magnetics, vol. 54, no. 8, Aug. 2018.

[2] T. Matsuzaki, M. Takemoto, S. Ogasawara, et al, "Novel Structure of Three-Axis Active-Control-Type Magnetic Bearing for Reducing Rotor Iron Loss," IEEE Transactions on Magnetics, vol. 52, no. 7, Jul. 2016.

[3] L. Ji, "Vibration mechanism analysis of magnetic levitation rotor system for low temperature waste heat power generation," Electric Machines and Control, vol. 23, no. 11, pp. 67-75, Nov. 2019.

[4] L. Ji, L. Xu and C. Jin, "Research on a Low Power Consumption Six-Pole Heteropolar Hybrid Magnetic Bearing," IEEE Transactions on Magnetics, vol. 49, no. 8, pp. 4918-4926, Aug. 2013.

[5] H. Gao and L. Xu, "Real-time Feed-Forward Force Compensation for Active Magnetic Bearings System Based on $\mathrm{H} \infty$ Controller," Chinese Journal of Mechanical Engineering, vol. 24, no. 1, pp. 58-66, Jan. 2011.

[6] Q. Chen, G. Liu and B. Han, "Unbalance vibration suppression for AMBs system using adaptive notch filter," Mechanical Systems and Signal Processing, vol. 93, pp. 136-150, Feb. 2017.

[7] M. Dasharatha, B. Rajendra Naik and N. S. S. Reddy, "Low power and area efficient FIR filter using adaptive LMS algorithm," International Conference on Communication and Signal Processing, Chennai, India, 2017.

[8] C. Peng, J. Sun, X. Song, et al, "Frequency Varying Current Harmonics Elimination for Active Magnetic Bearing System via Multiple Resonant Controllers," IEEE Transactions on Industrial Electronics, vol. 64, no. 1, pp. 517-526, Jan. 2017.

[9] P. Cui, D. Han, G. Zhang, et al, "Robust Odd Repetitive Controller for Magnetically Suspended Rotor System," IEEE Transactions on Industrial Electronics, vol. 66, no. 3, pp. 2025-2033, Mar. 2019.

[10] Z. Wang, C. Mao and C. Zhu, "Current Compensation Control of Multiple Frequency Vibrations of the Rotor in Active Magnetic Bearing High Speed Motors," Proceedings of the Csee, vol. 38, no. 1, pp. 275-284, Jan. 2018.

[11] C. Liu, and G. Liu, "Autobalancing Control for MSCMG Based on Sliding-Mode Observer and Adaptive Compensation," IEEE Transactions on Industrial Electronics, vol. 63, no. 7, pp. 4346-4356, Jul. 2016.

[12] K. Nonami, F. Qi-Fu, H. Ueyama. "Unbalance Vibration Control of Magnetic Bearing Systems Using Adaptive Algorithm with Disturbance Frequency Estimation," JSME International Journal Series $C$, vol. 41, no. 2, pp. 220-226, 1998.

[13] K. Jiang, C. Zhu and L. Chen, "Unbalance Compensation by Recursive Seeking Unbalance Mass Position in Active Magnetic Bearing-Rotor System," IEEE Transactions on Industrial Electronics, vol. 62, no. 9, pp. 5655-5664, Sep. 2015.
[14] C. Mao and C. Zhu, "Unbalance Compensation for Active Magnetic Bearing Rotor System Using a Variable Step Size Real-Time Iterative Seeking Algorithm," IEEE Transactions on Industrial Electronics, vol. 65, no. 5, pp. 4177-4186, May. 2018.

[15] Z. Wang, C. Zhu and L. Chen. "Parameter Design and Analysis for Two-degrees of Freedom Radial Hybrid Magnetic Bearings," (in Chinese) China Mechanical Engineering, vol. 38, no. 12, pp. 3699-3708, Jun. 2018.

[16] J. Shi, R. Zmood and L. Qin, "Synchronous disturbance attenuation in magnetic bearing systems using adaptive compensating signals," Control Engineering Practice, vol. 12, no. 3, pp. 283-290, Mar. 2004.

[17] T. Song, B. Han and S. Zhen, "Variable polarity LMS feedback based on displacement nulling to compensate unbalance of magnetic bearing," (in Chinese) Journal of Vibration \& Shock, vol. 34, no. 7, pp. 24-32, Jul. 2015.

[18] T. Schuhmann, W. Hofmann and R. Werner, "Improving Operational Performance of Active Magnetic Bearings Using Kalman Filter and State Feedback Control," IEEE Transactions on Industrial Electronics, vol. 59, no. 2, pp. 821-829, Feb. 2012.

[19] B. Han, Y. Liu and S. Zhen, "Research on Vibration Suppression for Magnetic Suspension Motor Based on Repetitive Control Method," (in Chinese) Journal of Vibration Measurement \& Diagnosis, vol. 35, no. 3, pp. 486-492, Jun. 2015.

[20] T. Song, B. Han and S. Zhen, "Variable polarity LMS feedback based on displacement nulling to compensate unbalance of magnetic bearing," (in Chinese) Journal of Vibration \& Shock, vol. 34, no. 7, pp. 24-32, Jul. 2015.

[21] C. Zhang, X. Zhao, Z. Guo, et al, "Three Improved Second Order Generalized Integrators and the Comparative Analysis in Phase Locked Loop Application," (in Chinese) Transactions of China Electrotechnical Society, vol. 32, no. 22, pp. 48-55, Nov. 2017.

\section{Biographical notes}

Li Ji, born in 1984, in 2014, he received his Ph.D. degree in engineering from Nanjing University of Aeronautics and Astronautics, Nanjing, China. His current research interests are in the configuration and control method for high speed magnetic levitation motors.

Tel: +86-0-15952029815; E-mail: jili198504@hdu.edu.cn

Xue-qing Ma, born in 1996, is currently an assistant professor at Hangzhou Dianzi University Information Engineering School, China. Her research interests include power electronics, motor control and deep learning neural networks.

E-mail: maxueqing@hdu.edu.cn

Zhen-min Chen, born in 1957, is Chairman of ZYEC-EML technologies Co., Ltd., China. Her research interests are in the design and manufacture of PMSM.

E-mail: czm@zyec.com 
Figures

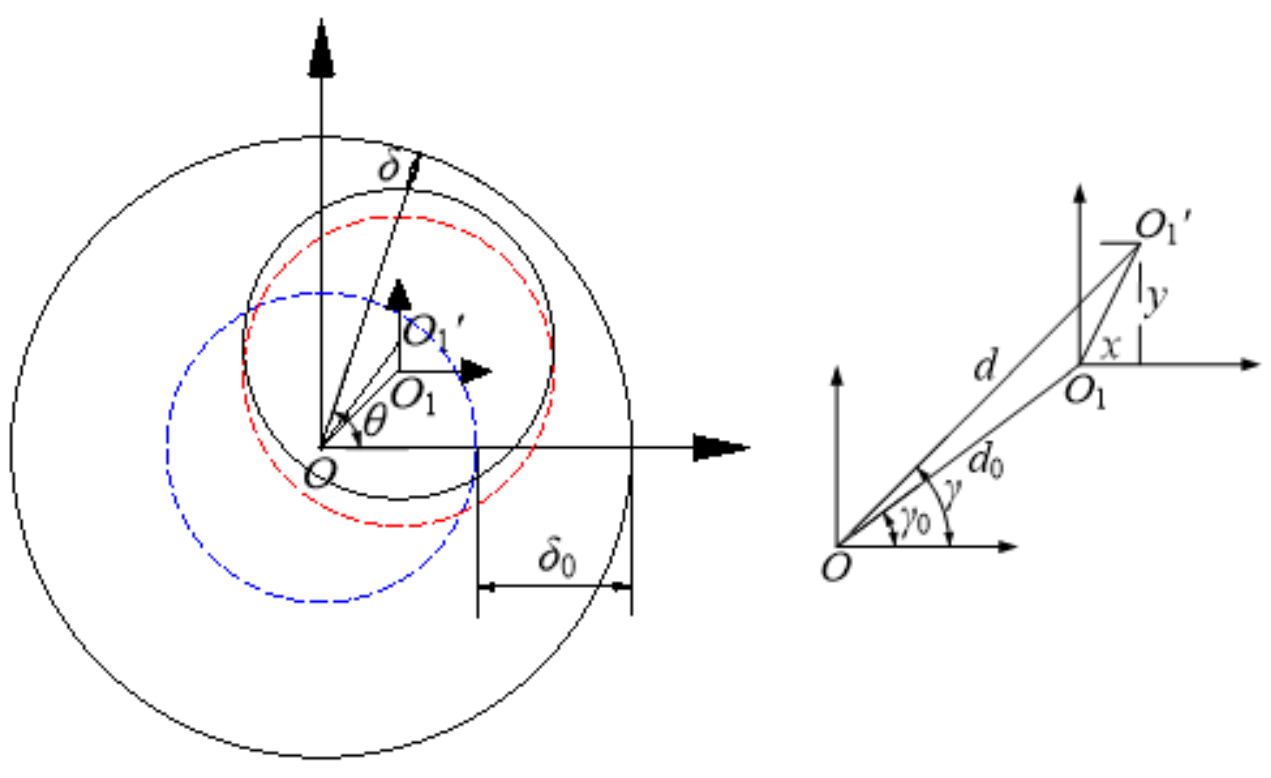

Figure 1

Cross-section of the maglev stator-rotor under air-gap eccentricity

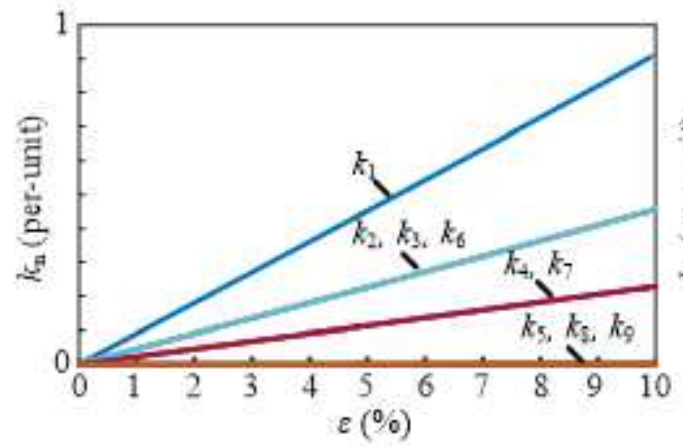

(a)

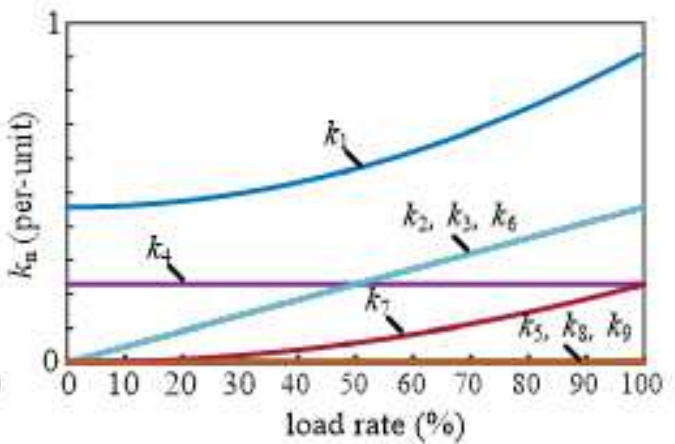

(b)

Figure 2

Unbalanced magnetic pull coefficient against eccentricity and load rate

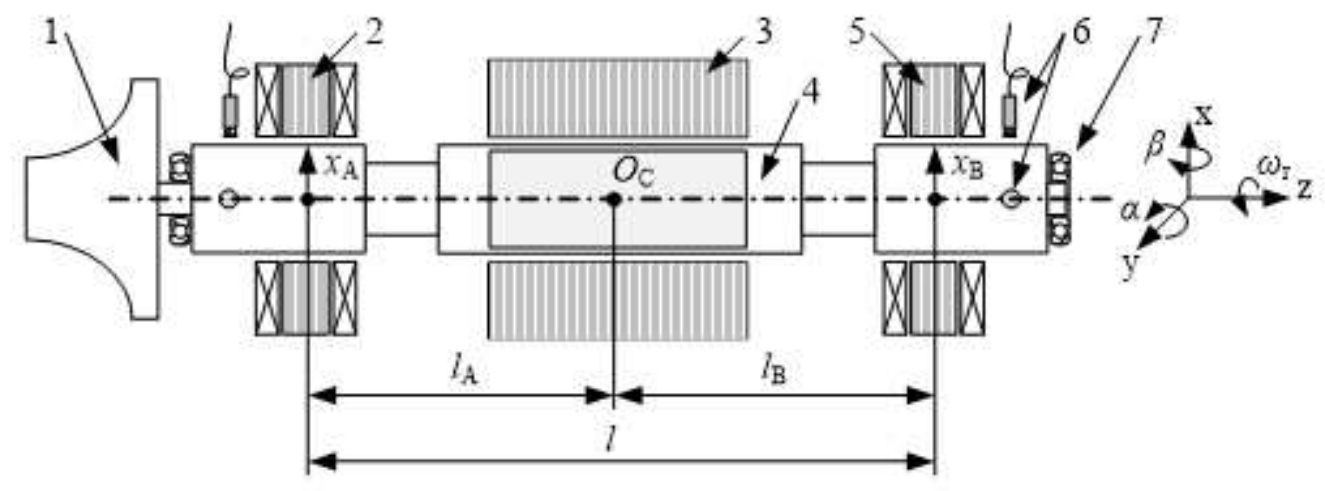


Figure 3

Schematic diagram of AMB-based motor. 1. Turbine impeller; 2. Radial magnetic bearing A; 3. Generator stator; 4. Maglev rotor; 5 . Radial magnetic bearing B; 6 . Radial displacement sensor; 7. Catcher bearing
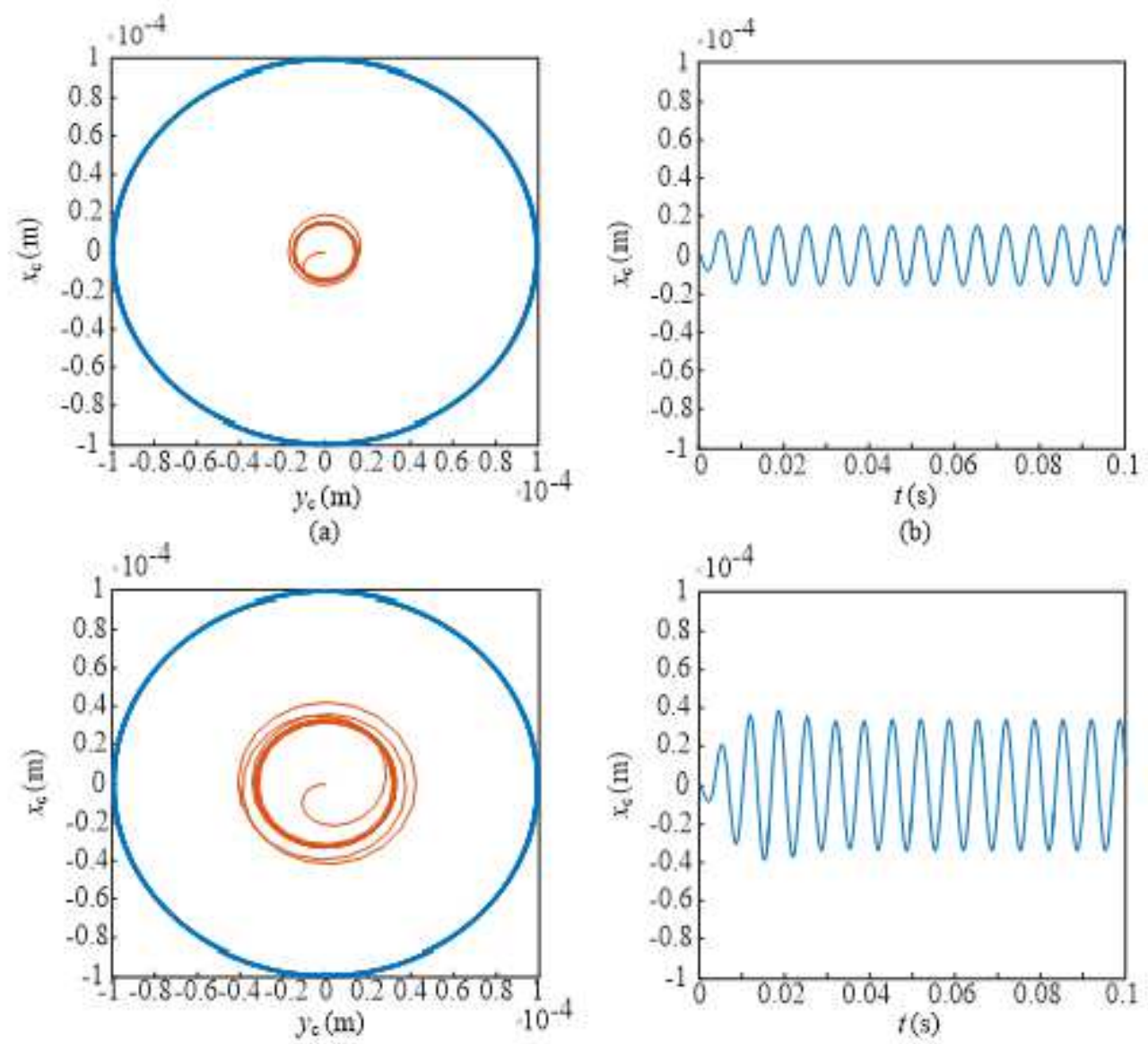

(c)

(d)

\section{Figure 4}

Simulation waveforms of rotor radial vibration with and without unbalanced magnetic pull

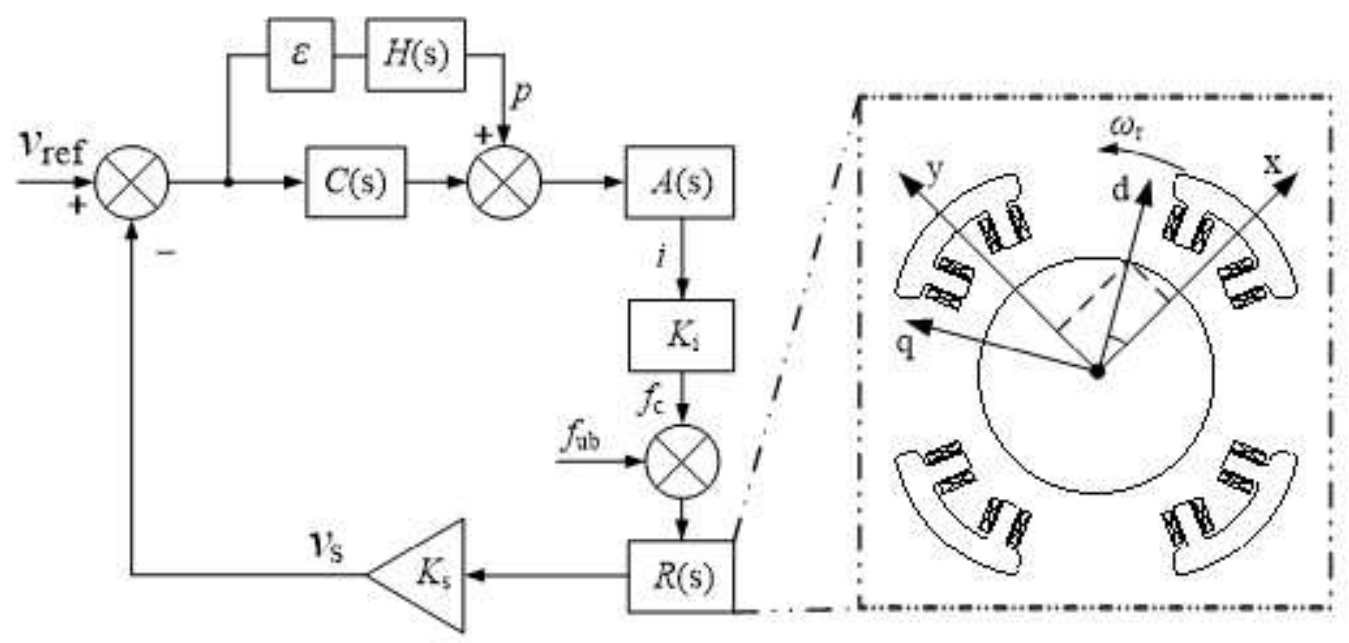

Figure 5 
The feedforward compensation control system of the magnetic bearing.

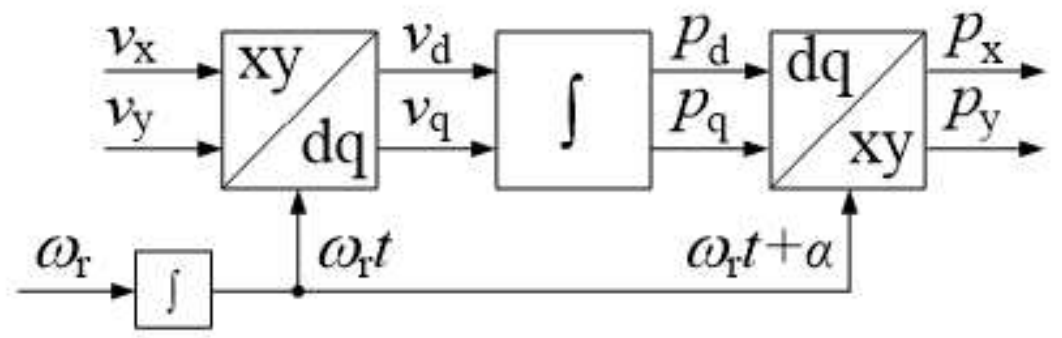

Figure 6

Structure diagram of HRF compensator.

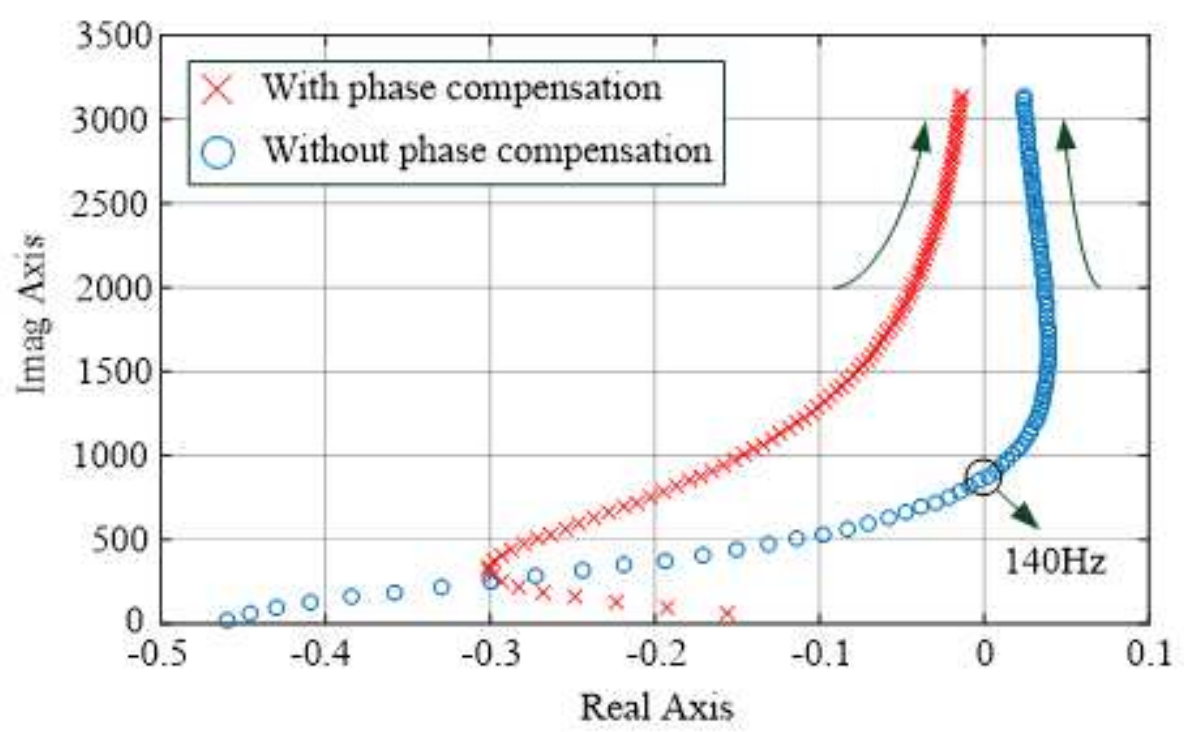

Figure 7

Dominant root locus of the control system with HRF compensator in simulation. 


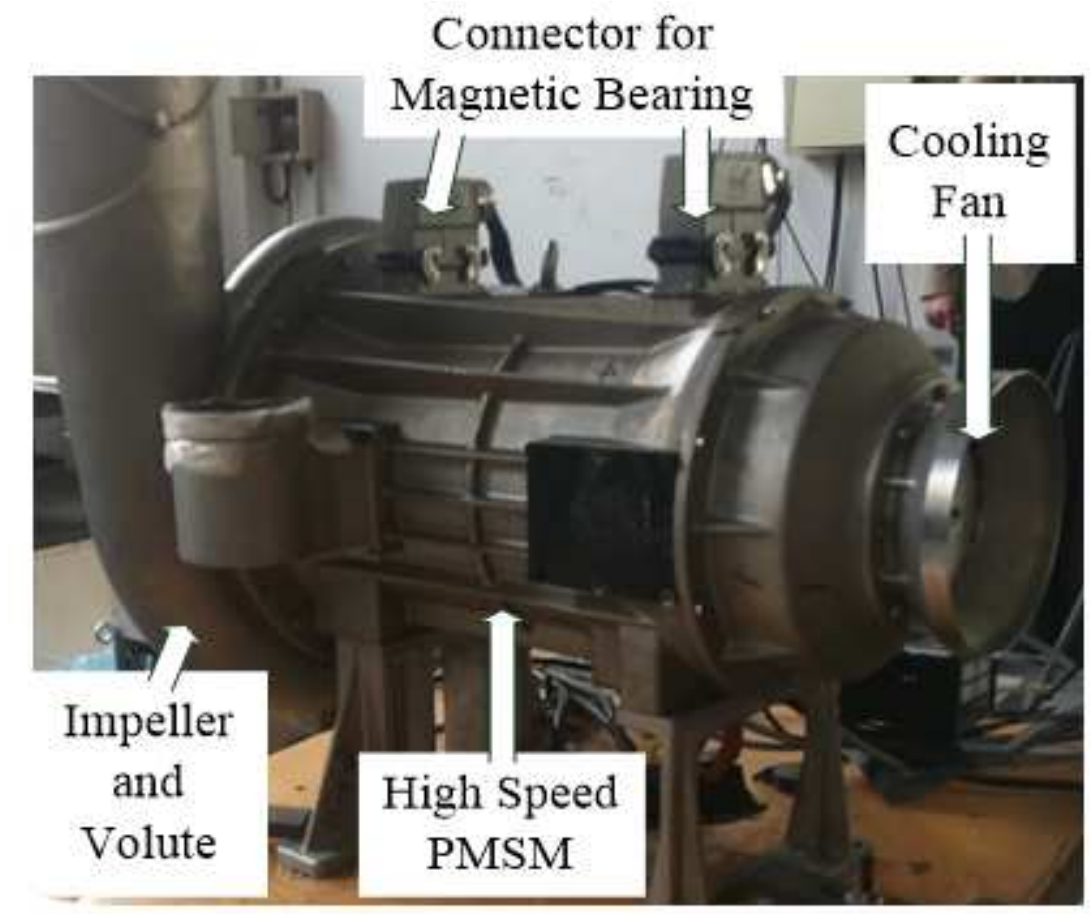

Figure 8

Functional prototype of the magnetic suspended PMSM.

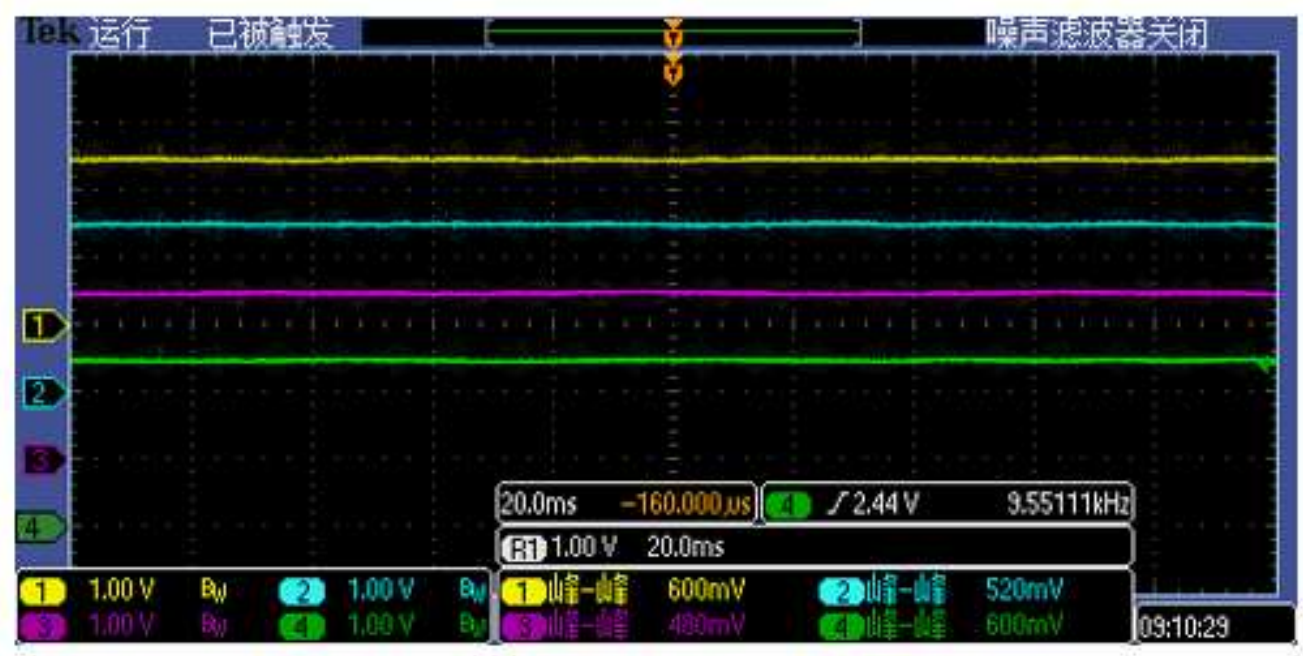

Figure 9

Experimental results of control system in steady state with the proposed method. 

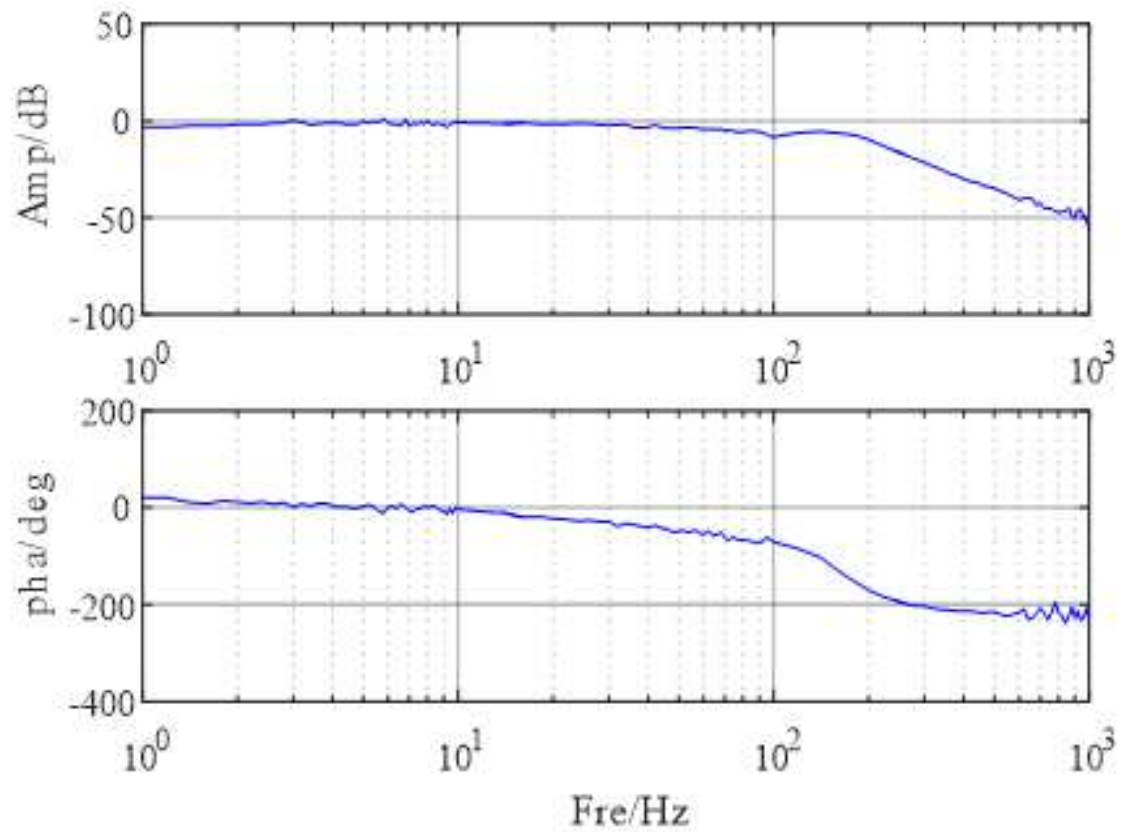

Figure 10

Closed-loop spectrum characteristics of the magnetic bearing-rotor system.

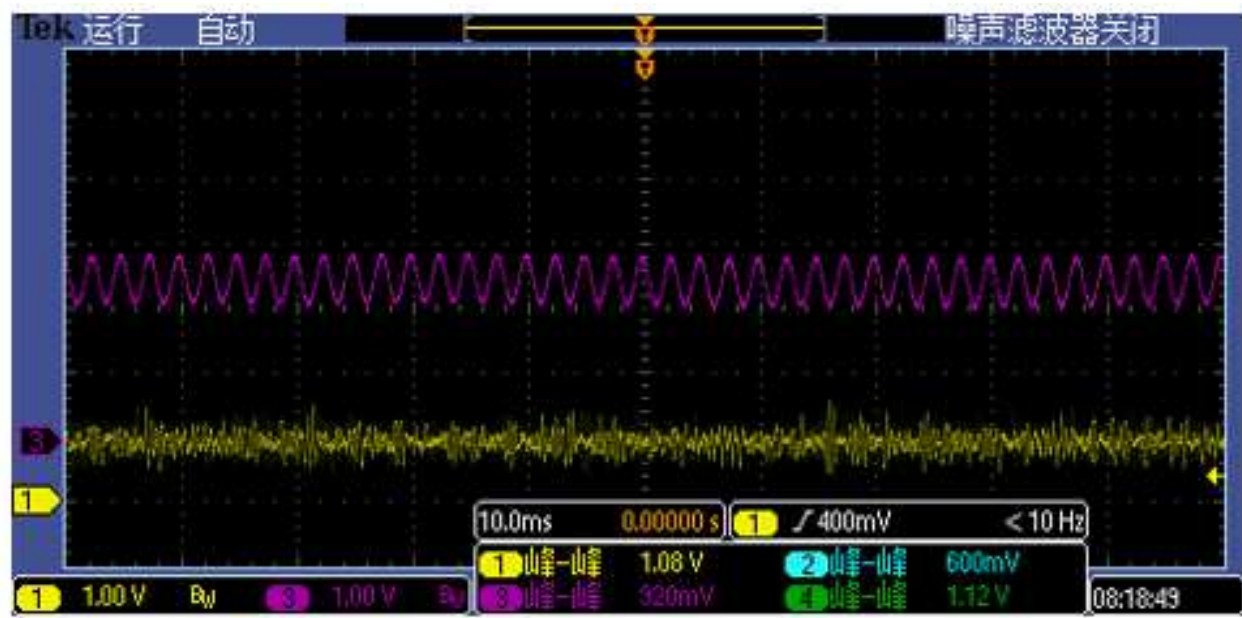

(a)

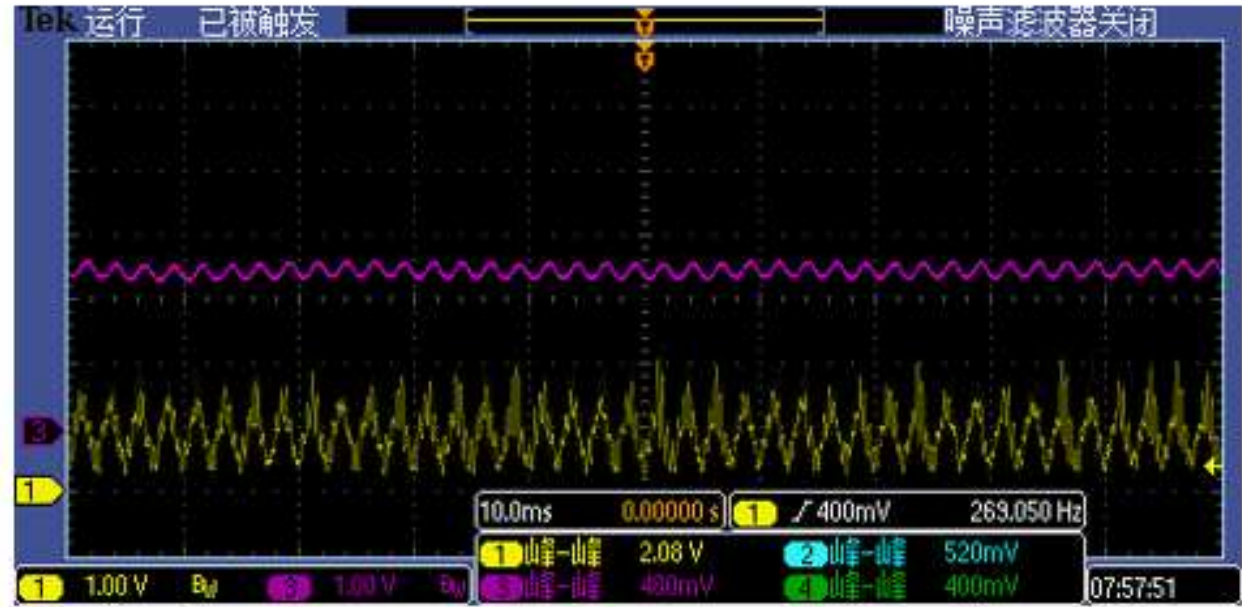

(b) 


\section{Figure 11}

Experimental results of magnetic bearing-rotor system with the compensator: (a) adaptive notch filter; (b) HRF compensator.

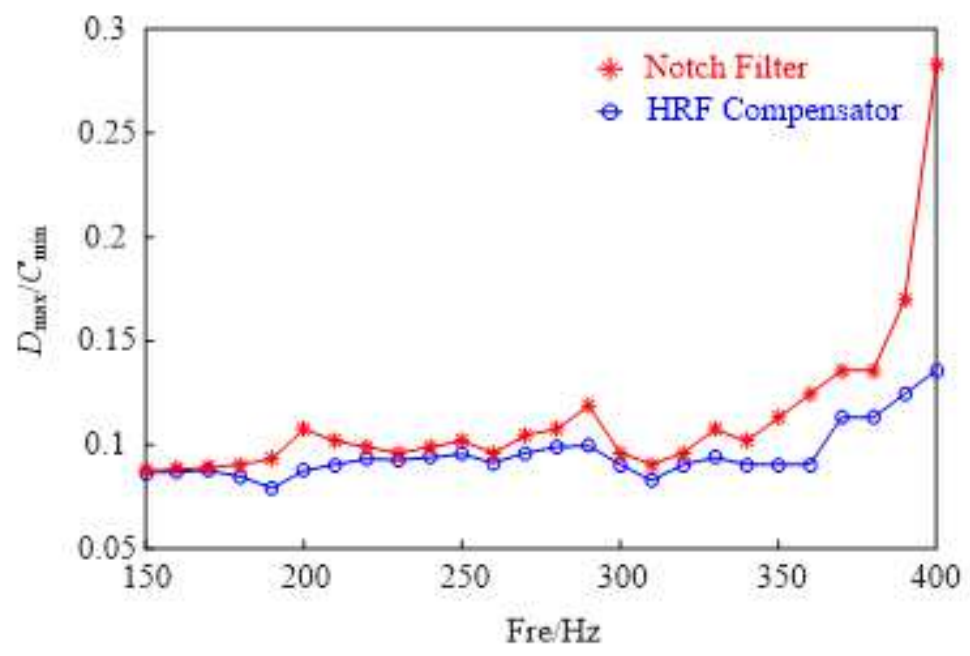

\section{Figure 12}

Vibration of the magnetic bearing-rotor system under different rotation speed. 